\title{
ANÁLISIS DE LAS PRODUCCIONES ESCRITAS DE UN JOVEN CON ALTAS CAPACIDADES DESDE LA PSICOLOGÍA POSITIVA
}

\author{
Fabiola Baquero Gomide \\ Universidad de Cantabria \\ Fbg911@alumnos.unican.es \\ Alba Ibañez García \\ Universidad de Cantabria \\ alba.ibanez@unican.es
}

Recepción Artículo: 13 octubre 2021

Admisión Evaluación: 13 octubre 2021

Informe Evaluador 1: 13 octubre 2021

Informe Evaluador 2: 14 octubre 2021

Aprobación Publicación: 14 octubre 2021

\section{RESUMEN}

La educación de las personas con altas capacidades tiene muchos conflictos e inconsistencias que pueden ser válidos y ricos para discusiones académicas, pero que también contribuyen a estimular barreras que impiden que estos estudiantes reciban reconocimiento educativo. De manera general esos estudios han avanzado en la búsqueda de la comprensión de los factores que, además de la inteligencia, son significativos en este proceso y resaltan el papel de los factores ambientales, sociales y emocionales en la expresión de habilidades superiores. Considerando la Psicología Positiva que estudia lo que hace que la vida merezca ser vivida y donde se estudia la experiencia óptima, esto es, a las personas siendo y dando lo mejor de sí mismas, se aborda un estudio de caso. Se plantea el análisis de 3 producciones de texto elaboradas por un joven con altas capacidades entre los 12 y 14 años, que participó en un programa de mentoría universitaria en Cantabria. En las producciones textuales se pudo observar la mejora en la calidad técnica de la escritura, más allá de la imaginación, la creatividad y la trascendencia como fortalezas que han evolucionado, integrado y asentado a rasgos personales al largo del tiempo. Se espera que los resultados del presente estudio puedan ampliar la comprensión de la dimensión emocional de estos individuos.

Palabras clave: altas capacidades; mentoría; psicología positiva

\section{ABSTRACT}

Analysis of the written productions of a gifted student from positive psychology. The topic of gifted education is found in many conflicts and inconsistencies that can be valid and rich for academic discussions, but that also contribute to stimulate barriers that prevent these students from receiving educational recognition. It is noted that, in spite of its importance, the socio-emotional characteristics of the gifted has received less 


\section{ANÁLISIS DE LAS PRODUCCIONES ESCRITAS DE UN JOVEN CON ALTAS CAPACIDADES DESDE LA PSICOLOGíA POSITIVA}

attention than the cognitive abilities and educational needs of gifted children in the literature of the field. Considering Positive Psychology as an approach to Psychology that studies what makes life worth living and where the optimal experience is studied, that is, people being and giving the best of themselves, a case study is approached. The analysis of 3 text productions made by a gifted male student between 12 and 14 years old, who participated in a university mentoring program in Cantabria, is proposed. In the textual productions it was possible to observe the improvement in the technical quality of the writing, beyond imagination, creativity and transcendence as strengths that have evolved, integrated and established personal traits over time. It is hoped that the results of the present study can broaden the understanding of the emotional dimension of these individuals.

Keywords: gifted; mentoring; positive psychology

\section{INTRODUCCIÓN}

El concepto de inteligencia y con ello el de altas capacidades - AC ha supuesto un cambio en los últimos años, desde una concepción psicométrica basada fundamentalmente en las puntuaciones obtenidas en los test de inteligencia, hacia modelos integradores en los que se tiene en cuenta no sólo la inteligencia sino otros factores vinculados a la personalidad, como la creatividad, la perseverancia en las tareas 0 la automotivación (Pfeiffer, 2015; Renzulli, 2015; Blumen, 2015).

En este sentido, también la atención educativa a las AC ha generado un cambio originado por las investigaciones que señalan que, aunque el desarrollo de las habilidades cognitivas es el aspecto que se enfatiza con mayor frecuencia, existen otros objetivos relacionados con la dimensión socioemocional que , siendo importantes, se les ha prestado mucha menos atención, como favorecer la ajuste personal y emocional de los superdotados, promover su desarrollo social y fortalecer una autoconcepto positivo (Alencar 2007, Alencar y Fleith, 2001; Renzulli, 2005, 2015; Subotnik et al., 2011, Dai 2013; Pfeiffer 2015), los aspectos relacionados con sus valores, sus actitudes y la motivación (Alencar y Fleith, 2001, Silverman et al., 2002).

La promoción del funcionamiento óptimo de los individuos observados entre las personas con AC ha sido examinada especialmente a la luz de la teoría de Csikszentmihalyi (1990), quien, junto a Seligman, lideran en las últimas décadas un movimiento denominado Psicología Positiva. El interés de esta corriente se centra en demostrar y reconocer que las virtudes y fortalezas personales actúan como agentes protectores y preventivos para la salud y el bienestar y que, por lo tanto, el conocimiento de tales fortalezas podría proporcionar lo que se ha llamado el florecimiento, es decir, "el vivir dentro de un rango óptimo de funcionamiento humano, que connota la existencia de bondad, generatividad, crecimiento y resiliencia" (Frederickson y Losada, 2005, p. 678), tanto de las personas como de comunidades e instituciones. Además, las virtudes y fortalezas personales están directamente influenciadas por las relaciones que el individuo establece a lo largo de su vida, ya sea en el ámbito familiar, social o cualquier otro entorno en el que interactúe, y la capacidad de reconocerlas y de ayudará a la persona en su capacidad para hacer cambios y ser resiliente. Peterson \& Seligman (2000) establecen 24 Fortalezas Personales que se engloban en 6 Virtudes humanas: sabiduría, valor, humanidad y amor, justicia, templanza y transcendencia, que son cualidades humanas que favorecerían una vida más plena y positiva.

Cuando se habla de la Psicología Positiva, no se debe de olvidar el peso que los valores tienen en el bienestar de la persona. Entre los temas de investigación de la corriente se encuentran el bienestar subjetivo, el optimismo, la resiliencia, las emociones positivas, las fortalezas de carácter y los talentos, la pasión, el sentido de vida y los valores (Tarragona, 2013). Csikszentmihalyi (1996), además, da un importante peso a la creatividad, considerada como un proceso clave para el desarrollo personal y para el progreso social y por ello se incluye de lleno dentro del campo de interés de la psicología positiva, entendiendo que puede ser considerada también como recurso para afrontar circunstancias adversas y como medio para resolver problemas, mejorando la calidad de la misma (Poseck, 2006).

Por todo ello, el principal objetivo de este estudio es centrarse en algunas dimensiones de ajuste y desarrollo socioemocional de alumnos con AC, desde el marco de las fortalezas humanas que la Psicología Positiva propone, ya que sugiere una visión más optimista y positiva del sujeto. 
En este sentido, para promover lo desarrollo de los alumnos con AC desde la comunidad educativa, se creó un programa de Mentorías Universitarias, Amentúrate, desde Facultad de Educación de la Universidad de Cantabria destinado a estudiantes con AC que cursen educación Secundaria Obligatoria en la comunidad de Cantabria. Desde este proyecto se plantea y define una propuesta innovadora en su diseño, con enorme valor educativo y creativo, así como responsable y sostenible. Contiene una primera parte de talleres o mentorías grupales, donde el alumnado conoce y participa en diversas áreas de conocimientos para, posteriormente, involucrarse en mentorías individuales, junto con un profesor de la universidad, en el que desarrollan un proyecto, producto 0 idea acorde con los intereses del alumno/a. Transversalmente se realizan otros talleres y actividades relacionados con el desarrollo socioemocional y de la creatividad. Su principal objetivo es mejorar el rendimiento académico del alumnado, ayudando a estos estudiantes a promover el sentimiento de autoeficacia, la percepción de un medio educativo favorecedor (Siegle y McCoach, 2013).

Se espera que el artículo presente contribuya a llamar la atención del público en general y de la comunidad educativa, para atender, en especial, a las características emocionales y sociales del alumnado con AC. Este aspecto es muy relevante debido a la necesidad de aportar medidas de apoyo a aquellos alumnos que destacan por su potencial superior, de tal manera que puedan lidiar mejor con su dimensión socioemocional.

Para ello, el siguiente artículo se centrará en un estudio de caso de un adolescente con AC que se destaca en el área intelectual. Esta comunicación es parte de un proyecto mayor que favorece la comprensión de fenómenos y valores ligados al bienestar y apoyo social percibido en alumnado con AC en un programa de mentoría universitaria.

\section{OBJETIVOS}

Comprender a través del análisis de producciones de texto las experiencias subjetivas positivas y rasgos individuales positivos de un joven con AC desarrolladas en un programa de mentoría universitaria.

\section{ESTRATEGIA METODOLOGICA - DESCRIPCIÓN DEL CASO}

Alex (nombre ficticio) es un chico que vive con su madre, su padre y hermano en Santander. Participó en el programa Amentúrate durante 2 años, entre 2019 y 2021, y tenía entre 12 y 14 años, reenviado por la Asociación Cántabra de Apoyo a las Altas Capacidades Intelectuales - ACAACI. Alex fue evaluado con AC aunque también presenta un perfil de talento artístico. Alex tiene la oportunidad de vivir en un espacio familiar saludable que favorezca su potencial, así como condiciones de una vida segura en cuanto a recursos materiales, educacionales y tecnológicos. Durante el período que participó en el programa, estuvo muy presente y activo. Participo en 10 talleres formativos, 3 talleres transversales y una experiencia de mentoría individualizada con un profesor de didáctica de la expresión artística de UC.

Para llevar a cabo el trabajo, se parte de una metodología cualitativa de análisis de producciones utilizando 3 textos generados por el alumno, buscando identificar los datos que permitan analizar sus rasgos individuales positivos y sus experiencias subjetivas positivas desarrolladas en Amentúrate (considerando el programa como una institución positiva).

Se comenzará con el análisis de la carta motivacional de 2019, pues este texto es un punto cero, es decir, su redacción no está influenciada por la participación en el programa, ya que se preparó antes de ingresar. De esta forma, podemos valorar sus motivaciones como expectativas previas y conocer rasgos individuales de Alex antes de las experiencias de mentoría universitaria.

El segundo texto para analizar es un texto de autorretrato, autodescripción escrito por Alex en diciembre de 2020, dirigido a un curso transversal denominado Arquitectura de Vida. Y finalmente, el tercer texto es un microrrelato, que redactó en 2021 para competir en un concurso de redacción promovido por la UC, y que resultó ganador. Es de destacar que las dos últimas producciones se realizaron durante el período de confinamiento debido a la pandemia del Covid 21 y fueron mediadas a través de sesiones virtuales. Alex produjo el material que se muestra aquí por sí mismo. 


\section{ANALISES Y DISCUSSION}

\section{Carta motivacional}

Para participar en el programa Amentúrate, Ios alumnos deben registrarse en el sitio web del programa. El proceso de selección se realiza analizando 3 cartas motivacionales: 1) del alumno; 2) de los padres; y 3) de un maestro 0 asesor 0 tutor escolar. Estos textos deben reconocer 0 describir las habilidades de los estudiantes, así como recomendar el programa por los beneficios que creen que puede aportarle (Gallego et al., 2009).

Para esta presentación sólo se analizará la carta motivacional de Alex, ya que permite establecer una reflexión sobre sus producciones personales y el desarrollo de su bienestar psicológico durante el período en el que participó en el programa. Esta es la carta motivacional:

"He conocido Amentúrate a través de mis padres. Me parece un programa muy interesante y una buena oportunidad para aprender cosas nuevas. He leído los talleres y a pesar de que me gustan varios, mi preferido es el de Astronomía. El tema del universo me apasiona, me parece increíble, y me gustaría aprender mucho más. Soy un niño que disfruto con nuevos retos y tengo muchas ganas de aprender. El colegio me aburre un poco, aunque este año he tenido un profesor genial que me ha propuesto investigar sobre temas que no conocía y he estado más motivado. Tengo muchas aficiones, pero sobre todo disfruto haciendo deporte, leyendo y jugando a la Play con mis amigos."

Peterson y Seligman (2004) empezaron a utilizar el concepto de fortaleza para referirse a la manifestación psicológica de la virtud, en otras palabras, las fortalezas humanas serían los ingredientes (procesos o mecanismos) psicológicos que definen las virtudes.

Entre los criterios de identificación de una fortaleza es de destacar el siguiente: "Una fortaleza debe manifestarse en la conducta de un individuo (pensamientos, sentimientos y/o acciones) de tal manera que pueda ser evaluada, tener cierto grado de generalibilidad en diferentes situaciones y estabilidad a lo largo del tiempo" (Giménez, 2010, p.39). A continuación, la Tabla 1 describe la taxonomía de Peterson y Seligman (2004).

Tabla 1.

Virtudes Humanas y Fortalezas Asociadas

\begin{tabular}{l|l}
\hline Virtud & Fortaleza Humana \\
\hline Sabiduría & Creatividad; Curiosidad/Interés; Deseo de aprender; \\
& Perspectiva; Apertura a la experiencia;
\end{tabular}

Valor $\quad$ Valor/valentia; Perseverancia; Vitalidad: Integridad;

\begin{tabular}{ll}
\hline Amor/humildad & Intimidad; Amabilidad; Inteligencia social; \\
\hline Justicia & Ciudadanía; Justicia; Liderazgo; \\
\hline Templanza & Capacidad de perdonar; Humildad; Prudencia; Autorregulación;
\end{tabular}

\begin{tabular}{ll}
\hline Transcendencia & Apreciación de la belleza; Gratitud; Optimismo Esperanza; \\
& Humor; Espiritualidad;
\end{tabular}

Al analizar la carta motivacional del alumno, es posible una relación entre la taxonomía presentada y el contenido de la carta motivacional. De esta forma, podemos identificar las fortalezas que se relacionan con la adquisición y el uso del conocimiento: desde la más básica desde el punto de vista evolutivo (curiosidad) hasta la más 
compleja (perspectiva). Y también fortalezas emocionales relacionadas a coraje y valentía con actuaciones conscientes dirigidas a objetivos encomiables, realizados ante fuertes adversidades y que no se sabe con certeza si serán conseguidos o no. Por ejemplo:

Me parece un programa muy interesante y una buena oportunidad para aprender cosas nuevas.

Soy un niño que disfruto con nuevos retos y tengo muchas ganas de aprender.

He leído los talleres y a pesar de que me gustan varios, mi preferido es el de Astronomía.

Los subrayados indican las fortalezas de la curiosidad, el interés, la búsqueda de lo nuevo y la apertura a la experiencia hacen referencia al propio deseo de experimentar y conocer de Alex. La curiosidad es el sistema emocional/motivacional positivo orientado hacia una forma alternativa de pensamiento, la actividad y la autorregulación de las experiencias e informaciones nuevas y cambiantes (Kashdan,2004).

Según los investigadores de la psicología positiva, tener ganas de aprender cosas nuevas es algo que puede estar presente a lo largo de toda la vida y una fortaleza importante asociada a salud mental y bienestar. Ahora bien, Giménez (2010) nos alerta que posiblemente sea en la infancia y la adolescencia, donde esta fortaleza juegue un papel más significativo por estar fuertemente relacionada con el contexto escolar. Además:

El colegio me aburre un poco, aunque este año he tenido un profesor genial que me ha propuesto investigar sobre temas que no conocía y he estado más motivado.

El tema del universo me apasiona, me parece increíble y me gustaría aprender mucho más.

Tengo muchas aficiones, pero sobre todo disfruto haciendo deporte, leyendo y jugando a la Play con mis amigos.

Los subrayados revelan las fortalezas de perseverancia y vitalidad de Alex. La perseverancia puede ser entendida como la persecución voluntaria de un objetivo a pesar de las dificultades y los obstáculos (Tice et al., 2004). Desde la psicología positiva se aborda la perseverancia, no como una obcecación, sino como la capacidad de tomar la decisión de cuándo seguir actuando y cuándo abandonar. En ese aspecto observamos que Alex (con 12 años) no tiene la opción de abandonar el colegio, pero se puede observar cómo persiste en continuar los estudios a pesar de los obstáculos (como el aburrimiento) buscando alternativas para alcanzar el objetivo final.

Vivir de forma apasionada y con energía, como si la vida fuera una aventura se refleja en la escritura de Alex. El implicarse en conocer, descubrir y tener ánimo para hacer las cosas. Ryan y Deci (2000) describen la vitalidad como un aspecto dinámico del bienestar relacionado con la experiencia subjetiva de energía y estar lleno de vida. Guarda relación tanto con una vitalidad física (encontrarse bien, sin fatiga ni enfermedad) como con una vitalidad psicológica (sentirse motivado, a gusto con uno mismo y con los demás, libre de tensiones, etc.).

Así, es posible observar que Alex presentaba, en el inicio del programa Amentúrate, rasgos individuales positivos con especial motivación en la búsqueda de conocimiento como objetivo principal para inscribirse en el programa de mentoría.

\section{Autorretrato \\ Mi autorretrato aquí presento, \\ que espero, no sea un tormento. \\ Por las mañanas soy como un perezoso, \\ pero nada risueño o bondadoso, \\ sino malhumorado y muy mosqueado, \\ cual toro toreado, en una plaza encerrado.}

En el taller Arquitectura de vida, se invitó a los estudiantes a crear un portafolio personal durante el transcurso de las 10 sesiones virtuales previstas para el año 2020/2021. En cada uno de ellos se propuso una actividad para: 0 promover el autoconocimiento; 0 la creatividad; 0 el área de interés de los alumnos. La primera actividad solicitada fue que los estudiantes se presentaran a través de un texto, un dibujo 0 una foto. Alex realizó las tres cosas: una foto, un dibujo y un poema. A continuación, el poema que lo describe: 
$Y$ es que todo, todito, me molesta, $y$, sin embargo, no tengo respuesta. Pero esto dura bien poco, Enseguida se me pasa, y soy otro. Como el Doctor Jekyll y el Señor Hyde ¿Es que en mi cuerpo habitan Bonnie y Clyde?

Me muevo más que las alas de un colibrí, Y soy tan testarudo como un jabalí. Amable y dócil como una suave brisa, Escandaloso cuando me da la risa

\author{
Ahora vamos con la prosopografía, \\ $Y$ con un poco de geografía; \\ Muy típica de España, \\ es mi piel tostada y aceitunada, \\ mis pestañas son negras y alargadas, \\ rasgos heredados de un bisabuelo de Málaga. \\ Se mezclan con mis lunares y mis pecas, \\ que son de unos antepasados belgas.
}

Para terminar, no quiero olvidar, que también tengo un apellido francés, Puestos a imaginar, quiero pensar, Que mi arte lo heredé de un pintor como Manet.

En este segundo texto, es de destacar la creatividad en su escritura. Si en 2019 Alex tenía ganas de aprender, demostraba intereses y apertura en experiencias, en 2020 manifestó su evolución a través de otro estilo de escritura. Así, desde una organización de pensamiento más básica que se observa en la producción de la carta motivacional, se valora, después de un año, una elaboración más compleja, organizada por rimas y estructuras de poema que se asocia a un domino específico de la lengua, como los versos, estrofas, rima, vocabulario, entre otros.

Abordar el tema de la creatividad no es nada fácil, entre otras cosas, porque no parece haber una definición clara del concepto (Amabile, 1983). De manera general, podemos afirmar que una persona creativa es aquella que produce ideas y conductas que son reconocidas como nuevas y originales y que tienen un valor adaptativo, es decir, que tiene repercusiones positivas para la propia persona o para los demás (Peterson y Seligman, 2004).

Otra fortaleza que se evidencia en esta escritura es, además de la creatividad y la autenticidad, los conceptos de integridad y honestidad que ayudan a definir como el rasgo que poseen las personas que se sienten seguras por ser como son, responsables de sus sentimientos y conductas, sinceras con sí mismas y con los demás (Sheldon et al., 2004). Según la psicología positiva la integridad presenta correlaciones con medidas psicológicas de bienestar como el ánimo positivo, la satisfacción con la vida, la apertura a la experiencia, la empatía, la auto-actualización y la responsabilidad (Ryan y Deci, 2000).

La evolución de las fortalezas de Alex identificadas en esta producción de su autorretrato demuestra que los adolescentes son capaces de idear alternativas y de imaginar nuevas soluciones, de enfrentarse a los problemas de una forma creativa y resiliente. 


\section{Microrrelato}

En 2021, se establece un concurso que une la literatura, las artes y la ciencia, incentivando la creatividad de Ios mentorizados, con la propuesta de construir un microrrelato que versase sobre la ciencia cotidiana, sobre la ciencia en el día a día con un número máximo de 150 palabras. Alex fue el primero a enviar su producto de escritura y fue también el ganador. Abajo su producción.

\section{UN DÍA MÁS}

Yotaro estaba deseando que acabase su jornada laboral. Su trabajo consistía en supervisar a los robots mientras estos fabricaban piezas para la industria automovilística. Pasaba allí ocho horas. Se le hacían eternas. Todos los días lo mismo. A las ocho, su coche inteligente le llevaba a su casa, también inteligente. Durante el trayecto observaba las calles, desoladas, ni un alma. Solamente coches y robots.

Llegó a casa.

- Bienvenido, Yotaro. ¿Qué tal el día? -le preguntó la casa.

Yotaro ni contestó, estaba harto de la misma conversación diaria.

- La cena está lista - dijo la casa.

Empezó a anochecer, las luces de la casa se regularon, las ventanas se volvieron opacas. Yotaro saboreaba la cena y se regocijaba pensando en que ya Ilegaban las diez, la hora de máximo disfrute. Terminó y se sentó en el sofá.

\section{-Esto es vida -exclamó Yotaro mientras se ajustaba sus gafas de realidad virtual}

En este tercer texto podemos observar la AC intelectual de Alex en función. Nuevamente se destaca su talento verbal, sus habilidades relacionadas con el lenguaje, su capacidad de comprensión, fluidez expresiva, dominio del vocabulario, aprendizaje de la lectura y escritura. También nos dan pistas sobre sus fortalezas personales para identificar a Alex como activo en la búsqueda de nuevos aprendizajes, en asumir riesgos en las propuestas, estar abierto a la experiencia, producir cosas nuevas. Tiene ganas de mejorar.

El programa Amentúrate se propone lograr con jóvenes de AC, experiencias subjetivas positivas, a partir de las ricas oportunidades de encuentros, diálogos y aprendizajes compartidos en las distintas mentorías grupales. Pues, cree que el aumento de las capacidades cognitivas y emocionales les van a permitir ser más conscientes de sus propias situaciones (revisar su pasado para conformar su presente y su futuro), adoptar diferentes puntos de vista (lo que le permitirá relacionarse con los demás de una manera diferente) y definir lo que son y lo que desean ser.

Sería precipitado afirmar que el programa fue responsable de este progreso, pero también no podemos dejar de mencionar su participación en este. Sabemos que las fortalezas individuales son constructos multidimensionales e influenciados por las condiciones contextuales, sociales y culturales.

\section{CONCLUSIONES}

En las producciones textuales de Alex se pudo observar la mejora en la calidad técnica de la escritura, más allá de la imaginación, la creatividad y la trascendencia como fortalezas que han evolucionado, integrado y asentado a rasgos personales al largo del tiempo. Podríamos, relacionar esta evolución con el concepto de Seligman (2011) que habla del "florecimiento humano" para referirse a las personas que funcionan a niveles extraordinarios en todos los ámbitos y que no solo se sienten bien, sino que hacen el bien, tienen un impacto positivo en su entorno. Alex en su poca edad deja huellas en sus textos de revelar su potencial, de 'aflorar' a través de este proceso de construcción y / la consolidación de su propia identidad. Estos textos nos animan en la medida en que sus capacidades cognitivas y emocionales pueden ayudarlo en un desarrollo pleno y feliz. Considerando que este artículo es parte de un proyecto de investigación más amplio las análisis aquí reportados deben entenderse como una construcción que no pretende proponer la última palabra, pero que tiene un carácter de síntesis provisional, abierta al análisis y a la producción permanente de conocimiento en la psicología positiva. Se espera que los 
resultados del presente estudio puedan ampliar la comprensión de la dimensión emocional de estos individuos. También se pretende que los resultados sirvan como subsidios para prácticas educativas que aborden el proceso de desarrollo del talento, así como impulsar lineamientos para programas de formación de docentes y psicólogos

\section{REFERENCIAS BIBLIOGRÁFICAS}

Alencar, E. M. L. S. (2007). Características sócio-emocionais do superdotado: questões atuais. Psicologia em Estudo, 12 (2), 371-378. https://doi.org/10.1590/S1413-73722007000200018

Alencar, E., \& Fleith, D. S. (2001). Superdotados: Determinantes, Educação e Ajustamento. Pedagógica e Universitária LTDA.

Amabile, T. M. (1983). The social psychology of creativity. Harcourt Brace.

Blumen, S. (2015). Abraham Tannenbaum: 1924-2014. Revista de Psicología (PUCP), 33(1), 231-234. https://revistas.pucp.edu.pe/index.php/psicologia/article/view/11177

Colangelo, N. \& Assouline, S. G. (2000, 2nd Ed.). Counselinggifted students. In K. A. Heller, F. J. Mönks, R. J. Sternberg \& R. F. Subotnik (Eds.), International handbook of research and development of giftedness and talent (pp. 595-608). Elsevier

Csiksentmihalyi, M. (1990). Flow: The psychology of optimal experience. Harper Collins.

Csikszentmihalyi, M. (1988). Motivation and creativity: Toward a synthesis of structural and energistic approaches to cognition. New Ideas in Psychology, 6, 159-176. https://doi.org/10.1016/0732-118X(88)90001-3

Dai, D. Y \& Chei, F. (2013), Three Paradigms of Gifted Education: In Search of Conceptual Clarity in Research and Practice. Gifted Child Quarterly 57(3)151-168 https://doi.org/10.1177/0016986213490020

Frederickson, B. L., \& Losada, M. F. (2005). Positive affect and the complex dynamics of human flourishing. American Psychologist, 60, 678-686. https://doi.org/10.1037/0003-066X.60.7.678

Gallego-Álvarez, T.; Ibañes-García, A.; Riva, J. A. la; Tororicagüena-Martín, M.; Villalón-Molina, R.; Ruiz-Cobo, R.; Guillén-Martín, V. (2019). Atención a las necesidades del alumnado con altas capacidades: Programa Amentúrate. En Consejo General de la Psicología IV Congreso Nacional de Psicología. 143-149.

Giménez, M. (2010). La medida de las fortalezas psicológicas en adolescentes (VIA Y): relación con clima familiar, psicopatología y bienestar psicológico. [Tesis doctoral Universidad Complutense, Madrid]

Giménez, M.,Vázquez, C., and Hervás, G.(2010). El análisis de las fortalezas psicológicas en la adolescencia: Más allá de los modelos de vulnerabilidad. Psychology, Society, \& Education, 2 (2), 97-116. https://dialnet.unirioja.es/servlet/articulo?codigo=3445211

Kashdan, T.B. \& Yuen, M. (2007). Wether highly curious students thrive aca- demically depends on perceptions about the school learning environment: a study of Hong Kong adolescents. Motivation and Emotion, 31, 260270. DOI 10.1007/s11031-007-9074-9

Peterson, C., \& Seligman, M. E. P. (2000). Values in action (VIA): Classification of strengths. Values In Action Institute.

Peterson, C. \& Seligman, M.E.P. (Eds.) (2004). Character strengths and virtues:a handbook and classification. American Psychological Association and Oxford University Press

Pfeiffer, S. I. (2015). El Modelo Tripartito sobre la alta capacidad y las mejores prácticas en la evaluación de los más capaces. Revista de Educación, (368), 66-95.

https://dialnet.unirioja.es/servlet/articulo?codigo $=5028336$

Poseck, B. V. (2006). Psicología positiva: una nueva forma de entender la psicología. Papeles del psicólogo, 27(1), 3-8.

Renzulli,J.S.(2005). The tree-ring conceptiona of giftedness: a developmental model for creative productivity. Em R.J . Sternerberg \& J. E.Davidson (Eds.), Conceptions of Giftedness (pp.246-279). Cambridge University Press. 
Renzulli, J. S., \& Gaesser, A. H. (2015). Un sistema multicriterial para la identificación del alumnado de alto rendimiento y de alta capacidad creativo-productiva. Revista de Educación, 368, 96-131. D0I: 10.4438/1988592X-RE-2015-368-290

Ryan, R.M. y Deci, E.L. (2000). Self-determination theory and the facilitation of intrinsic motivation, social development, and well-being. American Psychologist, 55, 68-78 D0I: 10.1037110003-066X.55.1.68

Seligman, M. E. (2011). Flourish: A visionary new understanding of happiness and wellbeing. Free Press.

Siegle, D. \& McCoach, D. B. (2013). Underachieving gifted students. In C. M. Callaham \& H. L. Hertberg- Davis (Eds.) Fundamentals of gifted education. Considering multiple perspectives (pp.377-387). Routledge

Silverman, L. K. (2002). Asynchronous development. In M. Neihart, S. M. Reis, N. M. Robinson \& S. M. Moon (Eds.), The social and emotional development of gifted children. Pufrock, 31-40.

Subotnik, R. F., Olszewski-Kubilius, P., \& Worrell, F. C. (2011). Rethinking Giftedness and Gifted Education: a Proposed Direction Forward Based on Psychologica Science. Psychological Science in the Public Interest, 12(1), 3-54. https://doi.org/10.1177/1529100611418056

Tarragona, M. (2013). Psicología positiva y terapias constructivas: una propuesta integradora. Terapia psicológica, 31(1), 115-125. http://www.redalyc.org/articulo.oa?id=78525710011

Tice, D.M., Wallace, H.M. y Harter, A.C. (2004). Persistence. En C. Peterson y M.E.P. Seligman (Eds.). Character strengths and virtues: a handbook and classification (pp. 229-247). American Psychological Association and Oxford University Press. 
